\title{
Compartilhamento de recursos por duas espécies de peixes nectobentônicas de riachos na bacia do rio Cachoeira (BA)
}

\author{
Maurício Cetra ${ }^{1,4}$, Giulianna Rodrigues Rondineli ${ }^{2} \&$ Ursulla Pereira Souza $^{3}$ \\ ${ }^{1}$ Curso de Ciências Biológicas, Universidade Federal de São Carlos - UFSCar, \\ Rod. João Leme dos Santos, Km 110, SP 264, CEP 18052-780, Sorocaba, SP, Brasil \\ ${ }^{2}$ Programa de Pós-graduação em Ciências Biológicas - Zoologia, Instituto de Biociências, \\ Universidade Estadual Paulista-UNESP, Av. 24 A, 1515, Bairro Bela Vista, CEP 13506-900, \\ Rio Claro, SP, Brasil \\ ${ }^{3}$ Departamento de Ecologia, Instituto de Biociências, Universidade Estadual Paulista - UNESP, \\ Av. 24 A, 1515, Bairro Bela Vista, CEP 13506-900, Rio Claro, SP, Brasil \\ ${ }^{4}$ Autor para correspondência: Mauricio Cetra,e-mail: mcetra@ufscar.br
}

CETRA, M., RONDINELI, G.R. \& SOUZA, U.P. Resource sharing by two nectobenthic freshwater fish species of streams in the Cachoeira river basin (BA). Biota Neotrop. 11(2): http://www.biotaneotropica.org. br/v11n2/en/abstract?article+bn01911022011.

\begin{abstract}
The aim of this study was to investigate specific characteristics and common features related to the abundance and the gradient of environmental conditions and food resources used by Trichomycterus bahianus and Characidium aff. zebra. These species are two typical streams fishes collected during the rainy season using electric fishing equipment in eight sites at the headwaters of the Cachoeira river basin, in a region of Atlantic rainforest in southeast of Bahia State. We quantified the correlation between environmental data and abundance through a principal component analysis. It was obtained the diet amplitude, the niche overlap and tested the differences in exploitation of food resources. In addition, we tested the differences in composition and degree of food preference in different stages of development. We collected 55 specimens of C. zebra and 124 of T. bahianus. It was found that the abundance of the two species is related to the gradient grass-rock on the shore and sand-boulder at the bottom. We can say that regardless of species, females were larger and heavier than males $(\mathrm{p}<0.001)$. The sex ratio of $C$. zebra differ from the expected $\left(\chi^{2}=4.091, \mathrm{df}=1, \mathrm{p}=0.043\right)$. The two species were classified as invertivorous, consuming mostly immature aquatic insects. There was no significant difference in the number of items consumed by the species, and the diet overlap was high $\left(\mathrm{O}_{\mathrm{i}, \mathrm{k}}=0.895\right)$. However, the species showed different strategies to obtain food which lead to significant differences in exploitation of food resources $(\mathrm{p}<0.001)$. This was evidenced when we took into account the different stages of development, showing that the diet of $C$. zebra is more generalist than T. bahianus. Probably, the sharing of abundant resources and differences in the occupation of microhabitats and life strategies allows the coexistence of this two species in the streams of the Cachoeira river basin during the rainy season.

Keywords: environmental conditions, diet, Atlantic rainforest, stage of development, Characidium zebra, Trichomycterus bahianus.
\end{abstract}

CETRA, M., RONDINELI, G.R. \& SOUZA, U.P. Compartilhamento de recursos por duas espécies de peixes nectobentônicas de riachos na bacia do rio Cachoeira (BA). Biota Neotrop. 11(2): http://www.biotaneotropica. org.br/v11n2/pt/abstract?article+bn01911022011.

Resumo: O presente estudo teve como objetivo buscar particularidades e aspectos em comum relacionados com as condições ambientais de ocorrência e recursos alimentares utilizados por Trichomycterus bahianus e Characidium aff. zebra, duas espécies típicas de riachos coletadas durante o período chuvoso na bacia do rio Cachoeira, sudeste da Bahia, uma região da Mata Atlântica, utilizando-se um equipamento de pesca elétrica em oito trechos de riachos de cabeceira. Foi verificada a correlação entre os dados ambientais e a abundância das espécies, através de uma análise de componentes principais. Para os itens alimentares verificou-se a amplitude da dieta, a sobreposição de nicho e a existência de diferenças na exploração dos recursos alimentares. Além disso, testou-se a existência de diferenças na composição e no grau de preferência alimentar em diferentes estágios de desenvolvimento. Foram coletados 55 exemplares de C. zebra e 124 de T. bahianus. Verificou-se que a abundância das duas espécies está relacionada com o gradiente gramínea-rocha na margem e areia-matacão no fundo. Pode-se afirmar que independente da espécie, as fêmeas foram maiores e mais pesadas que os machos ( $\mathrm{p}<0,001)$. A proporção sexual de $C$. zebra diferiu do esperado $\left(\chi^{2}=4,091 ; \mathrm{gl}=1 ; \mathrm{p}=0,043\right)$. As duas espécies foram classificadas como invertívoras, consumindo principalmente formas imaturas de insetos aquáticos. Não houve diferença significativa no número de itens consumidos pelas espécies. A sobreposição alimentar foi elevada $\left(\mathrm{O}_{\mathrm{j}, \mathrm{k}}=0,895\right)$, porém, as espécies apresentaram diferença significativa na exploração dos recursos alimentares ( $\mathrm{p}<0,001$ ). Observou-se também que, apesar da alta sobreposição alimentar, as espécies exploraram praticamente os mesmos recursos, porém, com estratégias distintas. Isto foi evidenciado quando se levou em consideração os 
diferentes estágios de desenvolvimento, mostrando que a dieta de C. zebra é mais generalista que a de T. bahianus. Provavelmente o compartilhamento de recursos abundantes e as diferenças na ocupação de micro-hábitats e nas estratégias de vida de C. zebra e T. bahianus, permita a coexistência das duas espécies nos riachos da bacia do rio Cachoeira durante o período chuvoso.

Palavras-chave: condições ambientais, dieta, Mata Atlântica, estágio de desenvolvimento, Characidium zebra, Trichomycterus bahianus.

\section{Introdução}

Compreender os limites impostos pelas condições ambientais às populações trata-se de um dos assuntos mais interessantes da Ecologia. Para cada grupo de organismos e ambientes nos quais ocorrem, existem particularidades características e, no caso dos peixes de riachos costeiros, as condições de instabilidade ambiental parecem assumir um papel estruturador fundamental, pois, ao regular o tamanho das populações (Winemiller 1989), influenciam na estruturação da comunidade (Langeani et al. 2005).

Em riachos de cabeceira, a disponibilidade de recursos, principalmente o alimentar, depende marcadamente do ambiente no entorno, que está constantemente fornecendo matéria orgânica e inorgânica (Allan 1995). No corpo d'água, devido ao transporte para jusante, somente uma fração reduzida de nutrientes toma parte nas interações ecológicas. De forma simplificada, pode-se dizer que os nutrientes inorgânicos na água passam para a forma orgânica na biota através, principalmente, de microorganismos. Uma vez na forma orgânica, irão passar pela teia trófica através de invertebrados e vertebrados pastadores. Sendo assim, os nutrientes ficarão fixados temporariamente em um determinado trecho do riacho. Através da decomposição irão retornar para a forma inorgânica deslocando-se rio abaixo, dando continuidade a um processo em espiral (Elwood et al. 1983).

A Mata Atlântica é um dos ecossistemas mais diversos e ameaçados do mundo. Riachos de grande correnteza e fundo rochoso normalmente constituem ambientes com elevada disponibilidade de alimento e áreas abrigadas, levando à ocorrência de espécies com adaptações morfológicas e comportamentais próprias, que contribuem para o maior sucesso nestes ambientes (Casatti \& Castro 1998, 2006).

Trichomycterus bahianus Costa, 1992 faz parte de um gênero de pequenos bagres com cerca de 100 espécies que medem entre 50 e $150 \mathrm{~mm}$ de comprimento padrão, ocorrem em riachos Neotropicais montanhosos, ficando restritas a pequenas áreas das bacias de drenagem e apresentam alto grau de endemismo (SarmentoSoares et al. 2005). Casatti \& Castro (2006) observaram que, de forma geral, as espécies pertencentes à família Trichomycteridae possuem corpo relativamente fino, alongado e deprimido, com barbilhões sensoriais bem desenvolvidos, olhos localizados no dorso, boca terminal ou subterminal e são nadadores pouco ativos. Para estes autores, estas características permitem a exploração de pequenos espaços entre as rochas, sendo caracterizados como bagres especuladores de substratos que se alimentam basicamente de larvas aquáticas de insetos, e classificados como nectobentônicos

Da mesma forma, Characidium aff. zebra Eigenmann, 1909 está dentro de um gênero rico, com cerca de 50 espécies que não ultrapassam $100 \mathrm{~mm}$ de comprimento padrão e ocorre em bacias hidrográficas Neotropicais (Graça \& Pavanelli 2008). Segundo Aranha et al. (2000) e Casatti \& Castro (2006), o corpo fusiforme e as nadadeiras peitorais e pélvicas alargadas, expandidas e localizadas na posição ventral, permitem a estas espécies manterem-se firmemente aderidas ao substrato, resistindo ao fluxo da corrente, caracterizandoas, também, como nectobentônicas. Habitam, preferencialmente, leitos pedregosos de trechos rápidos dos riachos, pois as características morfológicas favorecem a captura de alimento em locais de difícil acesso para outros peixes, como os pequenos espaços entre as pedras submersas, onde atuam como predadores de espreita alimentando-se de larvas aquáticas bentônicas de insetos (Aranha et al. 2000).

Characidium aff. zebra e T. bahianus são espécies características dos riachos de cabeceira da bacia do rio Cachoeira, no sudeste do estado da Bahia (Cetra et al. 2009), região com pouquíssimas informações biológicas a cerca de sua ictiofauna. Visando contribuir para o conhecimento da distribuição e abundância dessas duas espécies, o presente estudo fornece informações básicas sobre os recursos alimentares explorados e os efeitos das condições ambientais durante o período chuvoso na bacia do rio Cachoeira (BA). Para isso, procurou-se: i) estabelecer a relação entre a abundância das duas espécies com as características físicas, químicas e estruturais dos riachos; ii) classificá-las quanto à preferência alimentar; iii) verificar se há diferença na exploração dos recursos alimentares; e iv) avaliar a similaridade da dieta nos diferentes estágios de desenvolvimento.

\section{Material e Métodos}

\section{1. Área de estudo}

A bacia do rio Cachoeira está localizada na região centro-leste do estado da Bahia, entre as coordenadas $14^{\circ} 23^{\prime}$ e $15^{\circ} 36^{\prime} \mathrm{S}$, e $38^{\circ} 56^{\prime} \mathrm{e}$ $40^{\circ} 09^{\prime}$ O, inserida na Região de Planejamento e Gestão das Águas (RPGA) III - Leste (Superintendência..., Bahia 2007) (Figura 1). Está limitada ao norte pelas bacias dos rios de Contas e Almada, ao sul pelas bacias dos rios Pardo e Una, a oeste pela bacia do rio Pardo e a leste pelo Oceano Atlântico. A área de drenagem da bacia

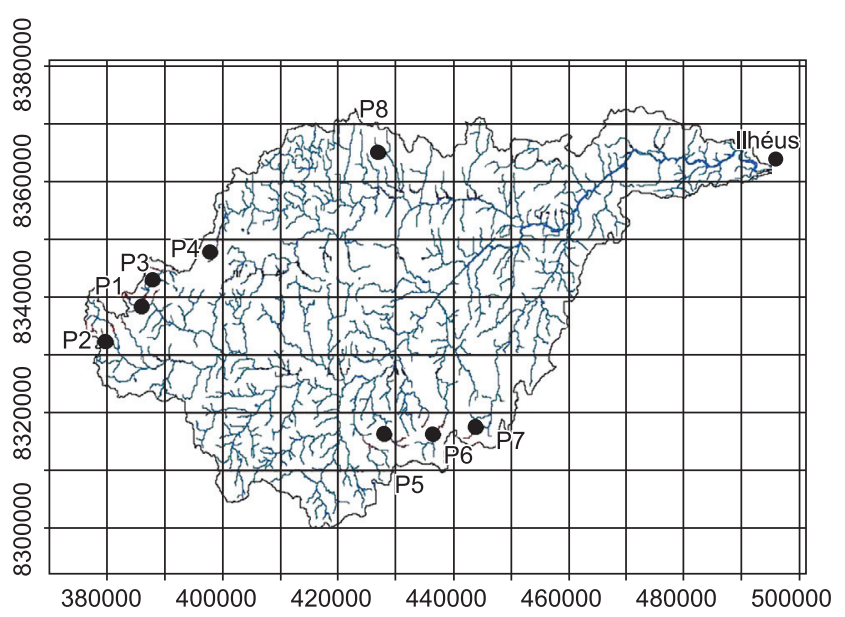

Figura 1. Localização geográfica da bacia hidrográfica estudada e pontos de coleta. Mapa da bacia do rio Cachoeira elaborado por Maurício Santana Moreau (DCAA/UESC).

Figure 1. Geographical location of the basin area and collection points. Map of Cachoeira river basin developed by Mauricio Santana Moreau (DCAA/UESC). 
corresponde a cerca de $4.600 \mathrm{~km}^{2}$ onde vivem aproximadamente 600.000 habitantes distribuídos em 12 municípios. As nascentes localizam-se na serra de Ouricana, numa altitude de $800 \mathrm{~m}$, na cidade de Itororó, com o nome de rio Colônia que, ao encontrar o rio Salgado, forma o rio Cachoeira que irá atingir seu patamar mais baixo no litoral de Ilhéus (Nacif et al. 2003).

A bacia do rio Cachoeira encontra-se inserida nas faixas climáticas do tipo Af, típico de florestas tropicais, com precipitação superior a $1.000 \mathrm{~mm}$ anuais, bem distribuída durante todo o ano e temperatura média de $24^{\circ} \mathrm{C}$. A zona climática, de transição, é do tipo Am, caracterizada pela ocorrência de um período seco nos meses de agosto a setembro, compensado pelos totais pluviométricos e temperaturas médias mensais elevadas e uma zona típica de clima tropical semi-úmido Aw, no sentido oeste próximo a região de planalto de Vitória da Conquista, com precipitação anual de $800 \mathrm{~mm}$ (Nacif et al. 2003).

\section{Coleta de dados}

Foi realizado um levantamento em oito riachos que compõem as regiões de cabeceira dos rios Colônia (pontos 1, 2, 3, 5 e 6), Piabanha (ponto 7) e Salgado (pontos 4 e 8) (Figura 1).

Os peixes foram coletados (Licença $n^{\circ}$ 13352-1 SISBIO/IBAMA/ MMA) durante o mês de dezembro de 2007 utilizando-se um aparelho de pesca elétrica. Um gerador com potência de $2.000 \mathrm{~W}$ foi conectado a um transformador de voltagem com entrada de $200 \mathrm{~V}$ e saída de até $500 \mathrm{~V}$. A este transformador foram ligados dois puçás que, dentro da água, forneciam uma corrente alternada máxima de $2 \mathrm{~A}$. A pesca elétrica foi realizada nos riachos em uma única vez no sentido jusantemontante em trechos de cerca de $70 \mathrm{~m}$ sem redes de contensão. Os organismos coletados foram imediatamente imersos em formaldeído a $10 \%$ para fixação e posteriormente transferidos para álcool a $70 \%$. Após a identificação, alguns indivíduos das espécies coletadas foram depositados no Museu de Zoologia da USP (MZUSP).

Antes do início da coleta da ictiofauna foram obtidos os valores de temperatura $\left({ }^{\circ} \mathrm{C}\right), \mathrm{pH}$ e condutividade $\left(\mathrm{mS} \mathrm{cm}^{-1}\right)$ utilizando-se uma sonda multiparâmetros Horiba U-10. Foram tomadas informações sobre largura, profundidade, velocidade de corrente e tipo de substrato. Após a coleta, o trecho amostrado foi subdividido em 10 segmentos transversais de $5 \mathrm{~m}$ nos quais foi medida a largura em centímetros. Em cada segmento transversal, da esquerda para a direita e de $1 \mathrm{em} 1 \mathrm{~m}$, foi anotada a profundidade em centímetros, a categoria de substrato predominante e a categoria de velocidade pontual da água na superfície. Independente do estado de preservação da vegetação ripária, a característica predominante do entorno foi classificada em margem com gramínea e composta por rochas. A porcentagem de cobertura vegetal foi graduada visualmente em quatro níveis: 1) 0 a $25 \%, 2) 26$ a $50 \%, 3) 51$ a $75 \%$ e 4) 76 a $100 \%$ (Cetra et al. 2009).

No laboratório foram tomados os seguintes dados de Characidium aff. zebra e Trichomycterus bahianus: comprimento total e padrão em centímetros, peso total em gramas, grau de repleção do estômago, grau de gordura acumulada na cavidade visceral, sexo e estádio de maturação gonadal (Braga 1990).

Ao grau de repleção estomacal foram atribuídos os seguintes valores: 1 para estômagos vazios, 2 para estômagos parcialmente cheios e 3 para estômagos completamente cheios. Os estômagos com grau de repleção igual a 3 foram retirados da cavidade visceral e pesados para posterior estudo da dieta.

Para o grau de gordura acumulada na cavidade visceral foram atribuídas três categorias: 1 para a cavidade visceral sem gordura, 2 para a cavidade visceral parcialmente preenchida com gordura e 3 para a cavidade visceral repleta de gordura.

O estádio de maturação gonadal de cada exemplar foi determinado macroscopicamente, quando possível, ou sob estereomicroscópio, considerando-se os seguintes aspectos: cor, transparência, vascularização superficial e, no caso de ovários, a visualização e aparência dos ovócitos. Ao estádio de maturação gonadal foram atribuídas quatro categorias (conforme escala previamente estabelecida): A para gônadas consideradas imaturas, B para gônadas em maturação ou em repouso, C para gônadas maduras e D para aquelas consideradas esgotadas (Vazzoler 1996).

\section{Análise dos dados}

Uma Análise de Componentes Principais (ACP) foi aplicada para simplificar e reduzir a dimensionalidade dos dados ambientais. Os escores dos eixos com autovalor superior a 1 foram correlacionados com a abundância das duas espécies utilizando o coeficiente de correlação de Pearson.

Após a identificação dos itens alimentares o método do GPA (Braga 1999) foi aplicado para classificá-los como preferencial absoluto (GPA igual a 4), alto grau de preferência (GPA entre 3 e 4), preferencial (GPA entre 2 e 3), secundário (GPA entre 1 e 2) e ocasional (GPA entre 0 e 1 ).

Foram verificadas a amplitude da dieta aplicando-se o índice de equabilidade de Pielou (J'), a sobreposição de nicho para as duas espécies através da aplicação do índice de Pianka $\left(\mathrm{O}_{\mathrm{i}, \mathrm{j}}\right)$ e foi testada a existência de diferença significativa na exploração dos recursos alimentares usando a técnica de modelos nulos com o algoritmo de aleatorização RA3, que mantém a amplitude de nicho e aleatoriza os zeros estruturais, conforme proposto por Gotelli \& Entsminger (2004).

Para os itens alimentares com GPA atribuído maior que 3, utilizando-se o índice de similaridade de Jaccard e de BrayCurtis foram aplicadas análises de similaridade (ANOSIM) com 10000 permutações para verificar se existe diferença significativa na composição das dietas e no grau de preferência alimentar, respectivamente, para as duas espécies em diferentes estágios de desenvolvimento foi aplicada uma técnica de análise multivariada de escalonamento multidimensional não-métrico (nmMDS) para os casos em que a ANOSIM resultou em diferença significativa (Hammer et al. 2001).

\section{Resultados}

Foram capturados 1.915 indivíduos pertencentes a cinco ordens, nove famílias e 26 espécies (Cetra et al. 2009). Destes, 55 indivíduos foram identificados como Characidium aff. zebra e 124 como Trichomycterus bahianus. As duas espécies ocorreram em cinco dos oito riachos amostrados e tiveram suas maiores capturas no ponto 6 , um riacho com largura média de $4 \mathrm{~m}$, profundidade de $21 \mathrm{~cm}$, velocidade superficial rápida, fundo com matacões, margem composta por cultura do cacau, temperatura em torno de $22{ }^{\circ} \mathrm{C}, \mathrm{pH}$ levemente ácido $(6,6)$ e condutividade de $70 \mathrm{mS} . \mathrm{cm}^{-1}$.

Os seis primeiros componentes principais que descrevem as condições físicas, químicas e estruturais dos riachos foram selecionadas através da ACP. No entanto, somente o segundo eixo (CPII) foi significativamente correlacionado com a abundância de T. bahianus $(\mathrm{r}=-0,80, \mathrm{n}=8, \mathrm{p}=0,02)$ e marginalmente significativo com C. zebra $(\mathrm{r}=-0,64, \mathrm{n}=8, \mathrm{p}=0,09)$. Este eixo foi responsável pela formação de um gradiente quanto ao tipo de fundo e margem. Esteve negativamente correlacionado com a ocorrência de margens com rocha $(r=-0,94)$ e fundos com grande porcentagem de matacões $(\mathrm{r}=-0,74)$ e positivamente relacionado com margens contendo gramíneas $(r=0,94)$ (Tabela 1). Como a correlação entre a abundância e o eixo também foi negativa, isto quer dizer que estas espécies ocorreram em maior número nos riachos com fundos formados por matacões com valores decrescentes de seixos e com margem composta por rochas e pouca gramínea (Figura 2). 
Tabela 1. Coeficientes para o segundo Componente Principal (CPII) da Análise de Componentes Principais baseada nos dados físicos, químicos e estruturais dos riachos de cabeceira da bacia do rio Cachoeira (BA).

Table 1. Second Principal Component (CPII) coefficients of the Principal Component Analysis based on data from physical, chemical and structural headwaters of the Cachoeira river basin (BA).

\begin{tabular}{lc}
\hline \multicolumn{1}{c}{ Variáveis ambientais } & CPII \\
\hline Margem de rocha* $(\%)$ & 0,46 \\
Fundo de matacão* $(\%)$ & 0,36 \\
Margem de gramínea* $(\%)$ & $-0,46$ \\
Velocidade rápida - V4 (\%) & 0,23 \\
Fundo de cascalho $(\%)$ & 0,21 \\
Cobertura vegetal & 0,17 \\
Largura média $(\mathrm{cm})$ & 0,12 \\
Velocidade lenta - V2 (\%) & 0,08 \\
Condutividade & 0,07 \\
Velocidade muito rápida - V5 $(\%)$ & 0,01 \\
Temperatura da água $\left({ }^{\circ} \mathrm{C}\right)$ & $-0,03$ \\
pH & $-0,04$ \\
Fundo de seixo $(\%)$ & $-0,11$ \\
Velocidade parada - V1 $(\%)$ & $-0,14$ \\
Fundo de material vegetal $(\%)$ & $-0,22$ \\
Velocidade moderada - V3 $(\%)$ & $-0,25$ \\
Profundidade média $(\mathrm{cm})$ & $-0,27$ \\
Fundo de areia $(\%)$ & $-0,29$ \\
\hline
\end{tabular}

*Variáveis significativamente correlacionadas com o segundo componente principal.

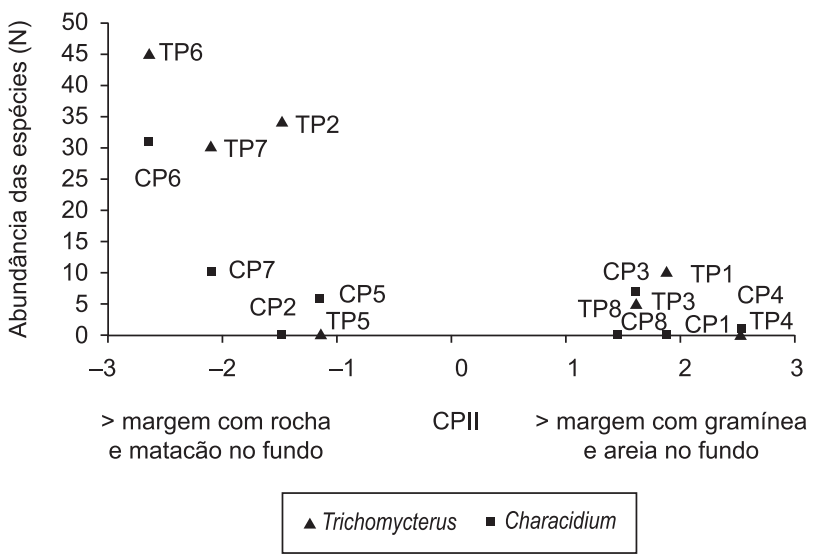

Figura 2. Correlação entre os escores do segundo componente principal (CPII) e os valores de abundância das espécies. Abreviações: CP - coordenadas do Characidium, TP - coordenadas do Trichomycterus, números de 1 a 8 pontos de coleta.

Figure 2. Correlation between the scores of the second principal component (PCII) and the values of species abundance. Abbreviations: CP - Characidium coordinates, TP - Trichomycterus coordinates, numbers 1 through 8 - the sites.

Os exemplares de $C$. zebra são menores e mais leves que os de $T$. bahianus $(\mathrm{p}<0,001)$ e, independente da espécie, as fêmeas foram maiores e mais pesadas que os machos $(\mathrm{p}<0,001)$. Todos os 20 machos de C. zebra estavam em maturação, as 35 fêmeas encontraram-se maduras e a proporção sexual diferiu do esperado $\left(\chi^{2}=4,091 ; \mathrm{gl}=1 ; \mathrm{p}=0,043\right)$. Dos 117 T. bahianus, 31 estavam em estádio imaturo, a maioria das fêmeas encontraram-se em maturação e a proporção sexual não diferiu do esperado $\left(\chi^{2}=2,977 ; \mathrm{gl}=1\right.$; $\mathrm{p}=0,084)$ (Tabela 2).

Poucos exemplares de C. zebra encontraram-se com estômago vazio e cavidade visceral repleta de gordura. Por outro lado, os indivíduos de T. bahianus distribuíram-se de forma igualitária nos três graus de repleção estomacal e a grande maioria estava com a cavidade visceral sem gordura (Tabela 3 ).

As duas espécies puderam ser classificadas como invertívoras, com os T. bahianus apresentando alto grau de preferência por larva de dípteros, através da utilização do GPA (Tabela 4). Qualitativa e quantitativamente as duas espécies não apresentaram diferenças significativas na dieta, pois o número de itens encontrados para ambas foi 10 e o valor do índice de equabilidade de Pielou foi $\mathrm{J}^{\prime}=0,71\left(\mathrm{IC}_{0,95}=0,69 \mathrm{a} 0,83\right)$ para $T$. bahianus e $0,84\left(\mathrm{IC}_{0,95}=0,69 \mathrm{a}\right.$ 0,86) para C. zebra. Apesar da elevada sobreposição alimentar, com índice de Pianka $\mathrm{O}_{\mathrm{j}, \mathrm{k}}=0,895$, as duas espécies apresentaram diferenças significativas quanto a exploração dos recursos alimentares $(\mathrm{p}<0,001)$.

Quanto à composição da dieta, não existiram diferenças significativas na similaridade dentro de cada espécie em seus distintos estágios de desenvolvimento e sexo. Tais diferenças foram encontradas na comparação entre machos de $C$. zebra em maturação e fêmeas de $T$. bahianus em maturação $(\mathrm{p}=0,047)$ e machos de C. zebra em maturação e machos de T. bahianus maduros $(\mathrm{p}=0,031)$. Dos oito itens alimentares, os machos de C. zebra utilizaram cinco, com frequência de ocorrência média entre 40 a $50 \%$ para três itens: larvas de Diptera, larvas de inseto e ninfa de Ephemeroptera. Por outro lado, nos estômagos de T. bahianus ocorreram sete itens, mas larva de Diptera ocorreu em todos os estômagos (100\%) e os outros itens foram pouco freqüentes (Tabela 5).

Os resultados da ANOSIM para a preferência alimentar foram semelhantes quanto a não existência de diferenças significativas na similaridade dentro de cada espécie em seus distintos estágios de desenvolvimento e sexo. Novamente, as diferenças foram encontradas na comparação entre machos de $C$. zebra em maturação e fêmeas de T. bahianus em maturação $(\mathrm{p}=0,001)$ e machos de $C$. zebra em maturação e machos de T. bahianus maduros $(\mathrm{p}=0,001)$. A preferência média refletiu o mesmo padrão encontrado para a composição (Tabela 6).

Adicionalmente, foram encontradas diferenças significativas na similaridade da preferência das fêmeas maduras de C. zebra e indivíduos imaturos de $T$. bahianus $(\mathrm{p}=0,046)$ e fêmeas maduras de C. zebra e machos maduros de T. bahianus ( $\mathrm{p}=0,021)$. Dos oito itens alimentares, as fêmeas dos Characidium utilizaram sete, com preferências médias maiores para larva de Diptera $(1,47)$ e ninfa de Plecoptera $(1,26)$. Por outro lado, nos estômagos dos imaturos de T. bahianus ocorreram somente quatro itens com preferência média em torno de três para larva de Diptera. Já para os machos maduros de T. bahianus também ocorreram sete itens, porém distintos daqueles encontrados nos de $C$. zebra, com preferência média acima de três para larva de Diptera (Tabela 6).

Através da visualização gráfica dos resultados gerados pelo escalonamento multidimensional não-métrico (nmMDS), tanto considerando composição da dieta (Figura 3a) ou preferência de itens (Figura 3b,c), percebeu-se que os dados dos itens alimentares de cada estômago de C. zebra mostraram-se mais distantes, demonstrando um padrão mais generalista que T. bahianus que, independente do estágio e sexo, foram mais próximos entre si (Figura 3).

\section{Discussão}

Ao se comparar a exploração dos recursos espaciais e alimentares pelas duas espécies em questão no período chuvoso, observou-se 
Tabela 2. Número de machos e fêmeas nos estádio em maturação (B) e maduro (C), comprimento médio (cm) e peso médio (g) com os respectivos erros padrão ( ) para as duas espécies.

Table 2. Number of males and females in maturation $(B)$ and mature $(\mathrm{C})$, length $(\mathrm{cm})$ and weight $(\mathrm{g})$ with their standard errors ( ) for both species.

\begin{tabular}{lcccc}
\hline & \multicolumn{2}{c}{ Characidium aff. zebra } & & \multicolumn{2}{c}{ Trichomycterus bahianus } \\
\cline { 2 - 5 } \cline { 4 - 5 } & Macho & Fêmea & Macho & Fêmea \\
\hline Maturação B & 20 & 0 & 21 & 30 \\
Maturação C & 0 & 35 & 31 & 4 \\
$\mathrm{C}_{\text {médio }}$ & $4,0(0,09)$ & $4,4(0,07)$ & $4,9(0,13)$ & $6,5(0,16)$ \\
$\mathrm{P}_{\text {médio }}$ & $0,9(0,10)$ & $1,5(0,07)$ & $1,3(0,18)$ & $3,0(0,22)$ \\
\hline
\end{tabular}

Tabela 3. Número de indivíduos com diferentes graus de repleção estomacal e de gordura acumulada na cavidade visceral para as duas espécies.

Table 3. Number of individuals with different degrees of repletion and fat accumulated in the visceral cavity of both species.

\begin{tabular}{lcccccc}
\hline & \multicolumn{3}{c}{ Repleção estomacal } & \multicolumn{3}{c}{ Gordura acumulada } \\
\cline { 2 - 7 } \cline { 4 - 7 } & $\mathbf{1}$ & $\mathbf{2}$ & $\mathbf{3}$ & $\mathbf{1}$ & $\mathbf{2}$ & $\mathbf{3}$ \\
\hline Characidium aff. zebra & 5 & 24 & 26 & 18 & 28 & 9 \\
Trichomycterus bahianus & 30 & 46 & 41 & 114 & 3 & 0 \\
\hline
\end{tabular}

Tabela 4. Valores de GPA para os diferentes itens alimentares encontrados nos estômagos de C. zebra e T. bahianus.

Table 4. Values of DFP for the different food items founded in the stomachs of C. zebra and T. bahianus.

\begin{tabular}{lc}
\hline \multicolumn{1}{c}{ Itens } & Characidium aff. zebra \\
\hline Ninfa Ephemeroptera & 0,839 \\
Ninfa Plecoptera & 0,935 \\
Larva Trichoptera & 0,806 \\
Larva Diptera & 1,387 \\
Larva de inseto & 0,469 \\
Isoptera & 0,806 \\
Inseto terrestre & 0,000 \\
Insetos (fragmentos) & 0,097 \\
Acarina & 0,032 \\
Crustacea & 0,194 \\
Material vegetal & 0,000 \\
Sementes & 0,041 \\
\hline
\end{tabular}

Tabela 5. Frequência de ocorrência média (\%) dos diferentes itens alimentares encontrados nos 12 estômagos dos diferentes estágios e sexos das duas espécies durante o período chuvoso nos riachos da bacia do rio Cachoeira (BA). (B: gônadas em maturação, C: gônadas maduras).

Table 5. Frequency of occurrence average (\%) of different food items found in the 12 stomachs of different stages and sexes of both species during the rainy season in the streams of the Cachoeira river basin (BA). (B: incomplete forms of gonad maturation, C: complete maturation forms).

\begin{tabular}{|c|c|c|c|}
\hline \multirow[t]{2}{*}{ Itens } & \multirow{2}{*}{$\begin{array}{c}\text { Characidium aff. zebra } \\
\text { Macho B }\end{array}$} & \multicolumn{2}{|c|}{ Trichomycterus bahianus } \\
\hline & & Fêmea B & Macho C \\
\hline Crustacea & - & 8,3 & 8,3 \\
\hline Larva Diptera & 58,3 & 100 & 100 \\
\hline Larva de inseto & 41,7 & 8,3 & 25 \\
\hline Larva Trichoptera & 16,7 & 50 & 8,3 \\
\hline Material vegetal & - & 8,3 & 8,3 \\
\hline Ninfa Ephemeroptera & 41,7 & 33,3 & 16,7 \\
\hline Ninfa Plecoptera & 16,7 & 16,7 & 8,3 \\
\hline
\end{tabular}


Tabela 6. Preferência alimentar média dos diferentes itens alimentares encontrados nos estômagos ( $\mathrm{n}=$ número de estômagos analisados) dos diferentes estágios e sexos das duas espécies durante o período de cheia nos riachos da bacia do rio Cachoeira (BA). (B: gônadas em maturação, C: gônadas maduras).

Table 6. Feeding preference average of the different food items found in the stomachs ( $\mathrm{n}=$ number of stomachs examined) of the different stages and sexes of both species during the rainy season in the streams of the Cachoeira river basin (BA). (B: incomplete forms of gonad maturation, C: complete maturation forms).

\begin{tabular}{|c|c|c|c|c|c|}
\hline \multirow[t]{2}{*}{ Itens } & \multicolumn{2}{|c|}{ Characidium aff. zebra } & \multicolumn{3}{|c|}{ Trichomycterus bahianus } \\
\hline & Fêmea C & Macho B & Imaturos & Fêmea B & Macho C \\
\hline Acarina & 0,32 & - & - & - & - \\
\hline Crustacea & - & - & - & 0,17 & 0,25 \\
\hline Larva Diptera & 1,47 & 1,25 & 2,91 & 3,00 & 3,33 \\
\hline Larva de inseto & 0,47 & 1,33 & 0,82 & 0,17 & 0,58 \\
\hline Larva Trichoptera & 0,95 & 0,58 & 0,18 & 0,75 & 0,17 \\
\hline Material vegetal & 0,11 & - & - & 0,25 & 0,08 \\
\hline Ninfa Ephemeroptera & 0,58 & 1,25 & 0,55 & 0,50 & 0,25 \\
\hline Ninfa Plecoptera & 1,26 & 0,42 & - & 0,17 & 0,08 \\
\hline $\mathrm{n}$ & 19 & 12 & 11 & 12 & - \\
\hline
\end{tabular}

que existem várias características comuns, como riacho de maior ocorrência, resposta ao gradiente ambiental, diferenças no tamanho e peso ao se comparar machos e fêmeas, estado de repleção estomacal, classificação trófica, quantidade, qualidade e similaridade na dieta dentro de cada espécie. Por outro lado, verificou-se a ocorrência de particularidades, como C. zebra ser menor e mais leve que T. bahianus, a proporção sexual de $C$. zebra ser diferente de 1:1 e a presença de imaturos de T. bahianus com ocorrência de maioria sem gordura acumulada na cavidade visceral. Observou-se também que, apesar da alta sobreposição alimentar, as espécies exploraram praticamente os mesmos recursos, porém, com estratégias distintas. Isto foi evidenciado quando se levou em consideração os diferentes estágios de desenvolvimento, mostrando que a dieta de C. zebra é mais generalista que a de T. bahianus. Como a distribuição geográfica de C. zebra é mais ampla, abrangendo as bacias do Orinoco, Amazonas e bacias costeiras no norte da América do Sul (Buckup et al. 2007) e a de T. bahianus é restrita à bacia do rio Una, Estado da Bahia (Pinna $\&$ Wosiacki 2003, Buckup et al. 2007), uma maior amplitude na dieta de $C$. zebra pode ter favorecido esta distribuição mais ampla.

A maior abundância de ambas as espécies ocorreu em um riacho com entorno formado por cabruca e o aumento no número de indivíduos responde ao gradiente de margem (gramínea-rocha) e de fundo (areia-matacão), ambientes propícios para espécies que ocorrem principalmente em trechos correntosos de riachos, com fundo rochoso (Casatti \& Castro 2006, Aranha et al. 2000). A cabruca é um sistema de cultivo agroflorestal (SAF), presente no sudeste baiano, em que ocorre a substituição do sub-bosque da Floresta Atlântica pelo cacaueiro Theobroma cacao. Apesar dos impactos causados por esta alteração estrutural e utilização de fertilizantes e pesticidas, pode-se considerar que este SAF permite a existência de relações mesológicas importantes para a conservação das espécies características da Mata Atlântica nesta região do Brasil (Faria et al. 2006). Neste sentido, a conservação de parte da vegetação no entorno evita a entrada de sedimentos no riacho, mantendo o hábitat das espécies em questão, além de permitir a ocorrência de espécies de invertebrados que têm parte do seu ciclo de vida dentro da água.

A variação no tamanho corpóreo é um dimorfismo sexual de ocorrência generalizada entre os peixes (Nikolsky 1963), sendo que o predomínio de fêmeas nas maiores classes de tamanho relaciona-se à seleção para o aumento da fecundidade (Nikolsky 1963, LoweMcConnell 1999). Para as duas espécies consideradas, as fêmeas foram maiores do que os machos. Resultados semelhantes foram encontrados para outras espécies de Characidium (Mazzoni et al.
2002, Braga et al. 2007, Becker et al. 2008). Casatti (2003) e Rondineli et al. (2009) encontraram machos maiores do que fêmeas em espécies de Trichomycterus.

Dados da proporção sexual podem fornecer importantes informações sobre a estrutura e dinâmica populacional. Characidium aff. zebra, nos riachos da bacia do rio Cachoeira, apresentou proporção sexual significativamente diferente do esperado (1:1), ocorrendo mais de uma fêmea por macho, enquanto que para T. bahianus o mesmo não ocorreu. Braga (2006) chama a atenção da importância em se conhecer a real segregação das populações estudadas, pois algumas podem distribuir-se melhor no ambiente em que vivem, provocando maior dispersão de machos e fêmeas, o que pode resultar no desvio da proporção esperada. Provavelmente, C. zebra distribui-se mais no ambiente em que vive do que $T$. bahianus, daí as diferenças encontradas nas proporções sexuais. Maior proporção de machos foi encontrada por Braga et al. (2007) e Mazzoni et al. (2002) em espécies de Characidium e Manriquez et al. (1988), Trajano (1997) e Rondineli et al. (2009) em espécies de Trichomycterus. O suprimento alimentar da população foi considerado por Nikolskii (1969) como fator importante na proporção sexual, constando que as fêmeas predominam quando o alimento disponível é abundante.

No trecho estudado na bacia do rio Cachoeira, as duas espécies foram classificadas como invertívoras, não sendo encontradas diferenças quantitativas e qualitativas na composição de suas dietas. Observou-se que a maioria dos itens consumidos foi de origem autóctone, sendo larvas de dípteros o principal item registrado, classificado como preferencial para T. bahianus. A preferência por larvas de Diptera foi verificada para Trichomycterus spp. no rio Passa Cinco (Rondineli et al. 2009), em um riacho no Parque Estadual do Morro do Diabo (Casatti 2002, 2003), em riachos no Chile (Habit et al. 2005) e na Colômbia (Chará et al. 2006). Espécies dos gêneros Characidium e Trichomycterus amostrados em riachos de Mata Atlântica foram classificadas como insetívoras, com preferência por insetos aquáticos, em diversos estudos (Sabino \& Castro 1990, Castro \& Casatti 1997, Aranha et al. 1998, Esteves \& Lobón-Cerviá 2001, Motta \& Uieda 2004, Castro et al. 2004, Barreto \& Aranha 2006, Esteves et al. 2008, Rondineli et al. 2009). A elevada participação de grupos cujas fases larvais são aquáticas ou semi-aquáticas demonstra a importância da contribuição dos insetos aquáticos para a ecologia dos sistemas terrestres adjacentes e para a própria dinâmica do corpo d'água, uma vez que parte desses indivíduos retorna para o sistema aquático (Roque et al. 2003). 
(a)
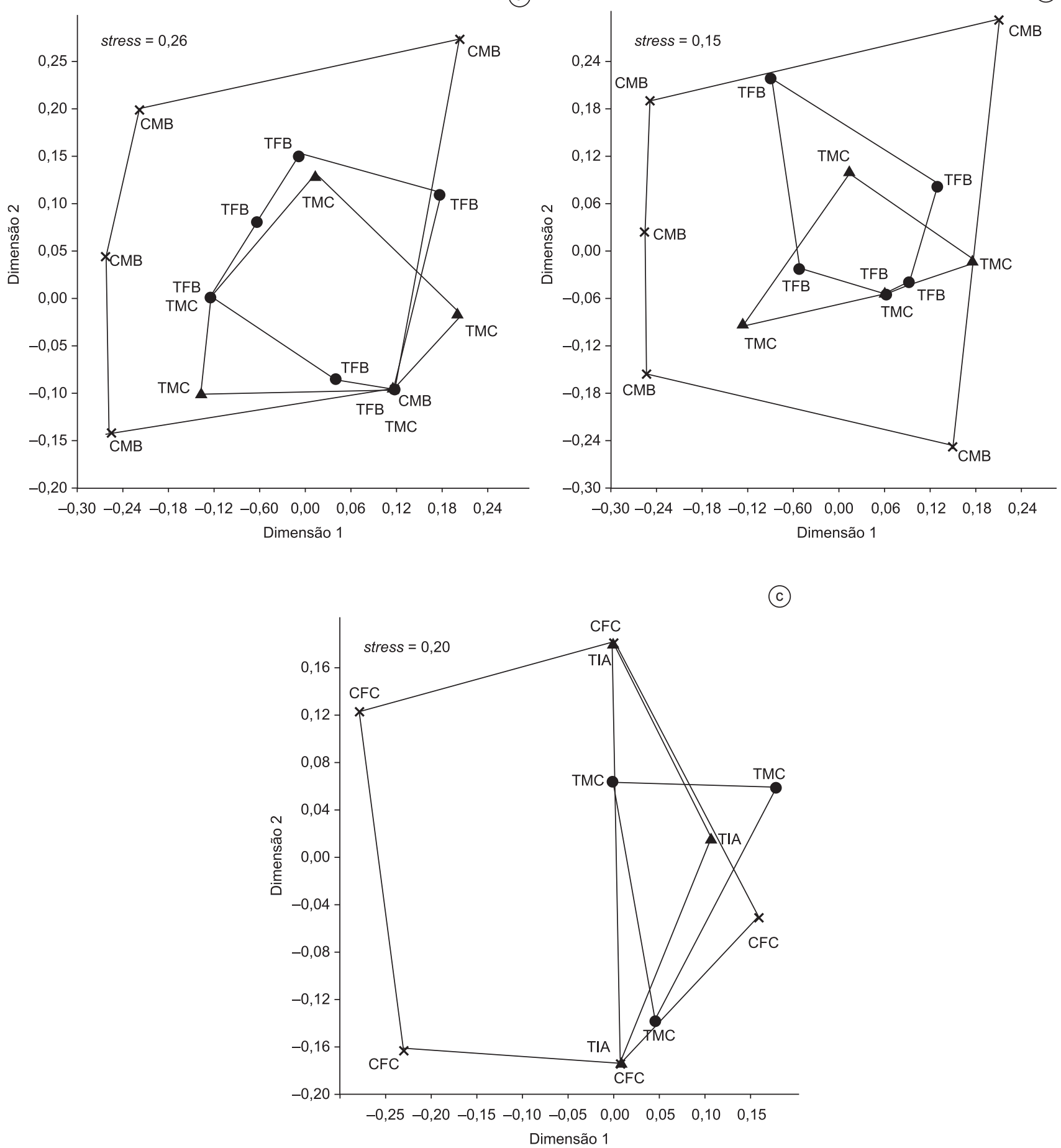

Figura 3. Projeção do escalonamento multidimensional não-métrico (nmMDS) e os menores polígonos convexos ("convex hulls") que encerram todos os dados pontuais dos estômagos das duas espécies nos diferentes estágios de maturação e sexo. a) índice de Jaccard; e b-c) índice de Bray-Curtis. Abreviações: Characidium macho no estágio de maturação B (CMB), Characidium fêmea no estágio de maturação C (CFC), Trichomycterus fêmea no estágio de maturação B (TFB), Trichomycterus macho no estágio de maturação C (TMC) e Trichomycterus imaturo (TIA).

Figure 3. Projection of non-metric multidimensional scaling (nmMDS) and the smaller convex polygons (convex hulls) that encapsulate all the data points from the stomachs of two species at different stages of maturation and sex. a) Jaccard index; and b-c) Bray-Curtis index. Abbreviations: Characidium male in the maturation stage B (CMB), Characidium female in the maturation stage C (CFC), Trichomycterus female in the maturation stage B (TFB), Trichomycterus male in the maturation stage $\mathrm{C}$ (TMC) and Trichomycterus immature (TIA). 
Characidium aff. zebra e T. bahianus apresentaram elevada sobreposição alimentar, mas houve diferença significativa na exploração dos recursos. A grande diversidade e variação na composição da comunidade de invertebrados aquáticos em riachos, ao longo do ano, pode ser a explicação para a extensa sobreposição na dieta dos peixes (Angermeier \& Karr 1983), mas a combinação de diferentes micro-hábitats, períodos de atividade e táticas utilizadas na captura do alimento podem minimizar os efeitos da sobreposição (Casatti 2002). Sabino \& Castro (1990) encontraram vários casos de alta sobreposição alimentar nas dietas de diferentes espécies e verificaram que as mesmas apresentavam diferenças nas distribuições espaciais e nos períodos alimentares. Pelo presente estudo, pode-se dizer que, além do que foi ressaltado anteriormente, tais diferenças estão representadas nos itens alimentares consumidos pelos distintos estágios de desenvolvimento, evidenciando que C. zebra mostrou-se mais generalista que o T. bahianus.

A sobreposição entre as dietas de Trichomycterus sp. e Characidium sp. foi verificada por Esteves \& Lobón-Cerviá (2001) em um riacho de Mata Atlântica, mas houve segregação espacial entre as espécies, com a primeira ocorrendo em locais mais profundos e a segunda em locais mais rasos. Na escala temporal, Casatti \& Castro (1998) observaram que as atividades de forrageio de duas espécies de Characidium foram diurnas, ao passo que, em Trichomycterus sp. foram predominantemente noturnas. Casatti (2002) amostrou espécies do mesmo gênero, foco do presente estudo, no fundo, entre rochas e classificou quanto às táticas alimentares, Trichomycterus sp. como especulador de substrato e Characidium sp. como predador de espreita. Barreto \& Aranha (2006) verificaram que C. lanei apresentou comportamento de localização, espreita e investida sobre o alimento, principalmente larvas, em um riacho de Mata Atlântica. Segundo Esteves \& Lobón-Cerviá (2001) e Esteves et al. (2008) o comportamento de "senta-e-espera", com captura das presas após inspeção visual, pode ser um mecanismo para evitar a competição.

Estudos que relacionam as características ambientais com alimentação, reprodução e abundância de espécies de peixes em riachos de Mata Atlântica, são fundamentais para a compreensão de como estes ambientes e suas comunidades estão estruturadas, fornecendo assim subsídios para trabalhos voltados à conservação e manejo de hábitats. Apesar da elevada sobreposição entre as dietas e maior abundância em locais estruturalmente semelhantes, o compartilhamento de recursos abundantes, as diferenças na ocupação de micro-hábitats, tanto na escala espacial quanto temporal, as distintas táticas alimentares e os itens alimentares explorados pelos distintos estágios de desenvolvimento das espécies parecem indicar uma possível explicação para a coexistência das espécies nos riachos da bacia do rio Cachoeira durante o período chuvoso.

\section{Agradecimentos}

Agradecemos à Profa. Dra. Erminda C.G. Couto, coordenadora do PPGSAT (UESC), à UESC pelo suporte logístico, ao CNPq (CTHIDRO 14/2005) pelo financiamento e aos Prof. MSc. Alberto L. Carmassi e Prof. MSc. Fábio C. Ferreira do PPCB (Zoologia UNESP/Rio Claro) pela coleta dos peixes.

\section{Referências Bibliográficas}

ALLAN, J.D. 1995. Stream Ecology: Structure and function of running waters. Chapman \& Hall, London.

ANGERMEIER, P.L. \& KARR, J.R. 1983. Fish communities along environmental gradients in a system of tropical streams. Environ. Biol. Fishes. 9(2):117-135. http://dx.doi.org/10.1007/BF00690857
ARANHA, J.M.R., GOMES, J.H. \& FOGAÇA, F.N.O. 2000. Feeding of two sympatric species of Characidium, C. lanei and C. pterostictum (Characidiinae) in a coastal stream of Atlantic Forest (Southern Brazil). Braz Arch Biol Tech. 43(5):527-531. http://dx.doi.org/10.1590/S151689132000000500013

ARANHA, J.M.R., TAKEUTI, D.F. \& YOSHIMURA, T.M. 1998. Hábitat use and food partitioning of the fishes in a coastal stream of Atlantic Forest, Brazil. Rev. Biol. Trop. 46(4): 951-959.

BARRETO, A.P. \& ARANHA, J.M.R. 2006. Alimentação de quatro espécies de Characiformes de um riacho da Floresta Atlântica, Guaraqueçaba, Paraná, Brasil. Rev. Bras. Zool. 23(3):779-788. http://dx.doi.org/10.1590/ S0101-81752006000300023

BECKER, F.G., CARVALHO, S. \& HARTZ, S.M. 2008. Life-history of the South American darter, Characidium pterostictum (Crenuchidae): evidence for small scale spatial variation in a piedmont stream. Neotrop. Ichthyol. 6(4):591-598. http://dx.doi.org/10.1590/S167962252008000400007

BRAGA, F.M.S. 1990. Aspectos da reprodução e alimentação de peixes comuns em um trecho do rio Tocantins entre Imperatriz e Estreito, Estado do Maranhão e Tocantins. Rev. Bras. Biol. = Braz. J. Biol. 50(3):547-558.

BRAGA, F.M.S. 1999. O grau de preferência alimentar: um método qualitativo e quantitativo para o estudo do conteúdo estomacal de peixes. Acta Sci. Biol. Sci. 21(2):291-295.

BRAGA, F.M.S. 2006. Aspectos da reprodução no gênero Characidium Reinhardt, 1867 (Crenuchidae, Characidiinae), na microbacia do Ribeirão Grande, serra da Mantiqueira, sudeste do Brasil. Acta Sci. Biol. Sci. 28(4):365-371.

BRAGA, F.M.S., SOUZA, U.P. \& CARMASSI, A.L. 2007. Dinâmica populacional de Characidium lauroi e C. alipioi (Teleostei, Crenuchidae) na microbacia do Ribeirão Grande, serra da Mantiqueira Oriental, Estado de São Paulo. Acta Sci. Biol. Sci. 29(3):281-287.

BUCKUP, P.A., MENEZES, N.A. \& GERAZZI, M.S.A. 2007. Catálogo das espécies de peixes de água doce do Brasil. Museu Nacional, Rio de Janeiro.

CASATTI, L. 2002. Alimentação dos peixes em um riacho do Parque Estadual Morro do Diabo, Bacia do Alto Rio Paraná, sudeste do Brasil. Biota Neotrop. 2(2):1-14.

CASATTI, L. 2003. Biology of a catfish, Trichomycterus sp. (Pisces, Siluriformes), in a Pristine stream in the Morro do Diabo State Park, Southeastern Brazil. Stud. Neotrop. Fauna E. 38(2):105-110. http://dx.doi. org/10.1076/snfe.38.2.105.15928

CASATTI, L. \& CASTRO, R.M.C. 1998. A fish community of the São Francisco Rivers headwaters riffles, southeastern Brazil. Ichthyol. Explor. Freshwaters. 9(3):229-242.

CASATTI, L. \& CASTRO, R.M.C. 2006. Testing the ecomorphological hypothesis in a headwater riffles fish assemblage of the rio São Francisco, southeastern Brazil. Neotrop. Ichthyol. 4(2):203-214.

CASTRO, R.M.C. \& CASATTI, L. 1997. The fish fauna from small forest stream of the upper Paraná River basin, southeastern Brazil. Ichthyol. Explor. Freshwaters. 7(4):337-352.

CASTRO, R.M.C., CASATTI, L., SANTOS, H.F., MELO, A.L.A., MARTINS, L.S.F., FERREIRA, K.M., GIBRAN, F.Z., BENINE, R.C., CARVALHO, M., RIBEIRO, A.C., ABREU, T.X., BOCKMAN, F.A., PELIÇÃO, G.A., STOPIGLIA, R. \& LANGEANI, F. 2004. Estrutura e composição da ictiofauna de riachos da bacia do rio Grande no Estado de São Paulo, sudeste do Brasil. Biota Neotrop. 4(1):1-39.

CETRA, M., FERREIRA, F.C. \& CARMASSI, A.L. 2009. Caracterização das assembléias de peixes de riachos de cabeceira no período chuvoso na bacia do rio Cachoeira (SE da Bahia, NE do Brasil). Biota Neotrop. 9(2):107-115. http://dx.doi.org/10.1590/S1676-06032009000200010

CHARÁ, JD., BAIRDS, DJ., TELFER, TC. \& RUBIO, EA. 2006. Feeding ecology and habitat preferences of the catfish genus Trichomycterus in low-order streams of the colombian Andes. Journal Fish Biol. 68(4):1026-1040. http://dx.doi.org/10.1111/j.0022-1112.2006.00984.x 
PINNA, M.C.C. \& WOSIACKI, W.B. 2003. Family Trichomycteridae. In Check list of the Freshwater Fishes of South and Central America. (R.E. Reis, S.O. Kullander, C.J. Ferraris, orgs.). EDIPUCRS, Porto Alegre, p. 270-290.

ELWOOD, J.W., NEWBOLD, J.D., O'NEILL, R.V. \& VAN WINKLE, W. 1983. Resource spiraling: an operational paradigm for analyzing lotic ecosystems. In: Dynamics of Lotic Ecosystem (T.D. Fontaine \& S.M. Bartell, eds.). Ann Arbor Science Publishers, Ann Arbor, p.3-28.

ESTEVES, K.E. \& LOBÓN-CERVIÁ, J. 2001. Composition and trophic structure of a fish community of a clear water Atlantic rainforest stream in southeastern Brazil. Environ. Biol. Fishes. 62:429-440. http://dx.doi. org/10.1023/A:1012249313341

ESTEVES, K.E., LOBO, A.V.P. \& FARIA, M.D.R. 2008. Trophic structure of a fish community along environmental gradients of a subtropical river (Paraitinga River, Upper Tietê River Basin, Brazil). Hydrobiologia. 598:373-387. http://dx.doi.org/10.1007/s10750-007-9172-4

FARIA, D., LAPS, R.R., BAUMGARTEN, J. \& CETRA, M. 2006. Bat and bird assemblages from forests and shade cacao plantations in two contrasting landscapes in the atlantic forest of southern Bahia, Brazil. Biodivers Conserv. 15(2):587-612. http://dx.doi.org/10.1007/s10531005-2089-1

GOTELLI, N.J. \& ENTSMINGER, G.L. 2004. EcoSim: Null models software for ecology. Version 7. Acquired Intelligence Inc. \& KeseyBear, Jericho. http://garyentsminger.com/ecosim/index.htm. (último acesso em 05/10/2009).

GRAÇA, W.J. \& PAVANELLI, C.S. 2008. Characidium heirmostigmata, a new characidiin fish (Characiformes: Crenuchidae) from the upper rio Paraná basin, Brazil. Neotrop. Ichthyol. 6(1):53-56.

HABIT, E., VICTORIANO, P. \& CAMPOS, H. 2005. Ecología trófica y aspectos reproductivos de Trichomycterus areolatus (Pisces, Trichomycteridae) en ambientes lóticos artificiales. Rev. Biol. Trop. 53(1-2):195-210.

HAMMER O., HARPER, D.A.T. \& RYAN, P.D. 2001. Past: Palaeontological statistics software package for education and data analysis. Palaeontol. Eletron. 4(1):9.

LANGEANI, F., CASATTI, L., GAMEIRO, H.S., CARMO, A.B. \& ROSSAFERES, D.C. 2005. Riffle and pool fish communities in a large stream of southeastern Brazil. Neotrop. Ichthyol. 3(2):305-311. http://dx.doi. org/10.1590/S1679-62252005000200009

LOWE-McCONNELL, R.H. 1999. Estudos ecológicos em comunidades de peixes tropicais. Edusp, São Paulo.

MANRIQUEZ, A., HUAQUIN, L., ARELANO, M. \& ARRATIA, G. 1988. Aspectos reproductivos de Trichomycterus areolatus Valenciennes, 1846 (Pisces: Teleostei: Siluriformes) en Rio Angostura, Chile. Stud. Neotrop. Fauna E. 23:89-102. http://dx.doi.org/10.1080/01650528809360749
MAZZONI, R., CARAMASCHI, E.P. \& FENERICH-VERANI, N. 2002. Reproductive biology of Characidiinae (Psteichthyes, Characidae) from the Ubatiba river, Maricá-RJ. Braz. J. Biol. 62(3):487-494. http://dx.doi. org/10.1590/S1519-69842002000300013

MOTTA, R.L. \& UIEDA, V.S. 2004. Dieta de duas espécies de peixes do ribeirão do Atalho, Itatinga, SP. Rev. Bras. Zoociências. 6(2):191-205.

NACIF, P.G.S., COSTA, L.M., SOADI, A., FERNANDES-FILHO, E.I., KER, J.C., COSTA, O.V. \& MOREAU, M.S. 2003. Ambientes naturais da bacia hidrográfica do rio Cachoeira. www.corredores.org.br/?pageId=adminO penDoc\&docId=1664 (último acesso em 14/04/2009).

NIKOLSKY, G.V. 1963. The ecology of fishes. Academic Press, London.

NIKOLSKII, G.V. 1969. Theory of Fish Population Dynamics: as the Biological Background for Rational Exploitation and Management of Fishery Resources. Oliver and Boyd Ltda, Edinburgh.

RONDINELI, G.R., CARMASSI, A.L. \& BRAGA, F.M.S. 2009. Population biology of Trichomycterus sp. (Siluriformes, Trichomycteridae) in Passa Cinco stream, Corumbataí River sub-basin, São Paulo State, southeastern Brazil. Braz. J. Biol. 69(3):925-934. http://dx.doi.org/10.1590/S151969842009000400022

ROQUE, F.O., PAPINELLI, M., FRAGOSO, E.N., FERREIRA, W.A., BARILLARI, P.R., YOSHINAGA, M.Y., TRIVINHO-STRIXINO, S., FENERICH-VERANI, N. \& LIMA, M.I.S. 2003. Ecologia de macroinvertebrados, peixes e vegetação ripária de um córrego de primeira ordem em região de Cerrado, do estado de São Paulo (São Carlos, SP). In: Ecótonos nas Interfaces dos Ecossistemas Aquáticos (R. Henry, org.) Editora Rima, São Paulo.

SABINO, J. \& CASTRO, R.M.C. 1990. Alimentação, período de atividade e distribuição espacial dos peixes de um riacho da Floresta Atlântica (Sudeste do Brasil). Rev. Bras. Biol. = Braz. J. Biol. 50(1):23-36.

SARMENTO-SOARES, L.M., MARTINS-PINHEIRO, R.F., ARANDA, A.T. \& CHAMON, C.C. 2005. Trichomycterus pradensis, a new catfish from southern Bahia coastal rivers, northeastern Brazil (Siluriformes: Trichomycteridae). Ichthyol. Explor. Freshwaters. 16(4):289-302.

SUPERINTENDÊNCIA DE RECURSOS HÍDRICOS - SRH \& Bahia (Estado). 2007. Região de planejamento e gestão das águas. Disponível em: http://www.srh.ba.gov.br/ (último acesso em 05/05/2007).

TRAJANO, E. 1997. Population ecology of Trichomycterus itacarambiensis, a cave catfish from eastern Brazil (Siluriformes, Trichomycteridae). Environ. Biol. Fishes. 50:357-369. http://dx.doi.org/10.1023/A:1007366119261

VAZZOLER, A.E.A.M. 1996. Biologia da reprodução de peixes teleósteos: teoria e prática. EDUEM, Maringá.

WINEMILLER, K.O. 1989. Patterns of variation in life history among South American fishes in seasonal environments. Oecologia. 81:225-241.

Recebido em 20/01/2010

Versão reformulada recebida em 08/06/2010

Publicado em 11/05/2011 